\title{
Pesquisa de matérias estranhas e avaliação físico-química de caldo-de-cana comercializado na região de Sete Lagoas - MG
}

\author{
Ducilaine Eduardo Rodrigues ${ }^{1}$, Clarissa Ane Gonçalves ${ }^{2}$, Larissa Barbosa Silva ${ }^{3}$, Luana Sousa Silva ${ }^{4}$, Andréia \\ Marçal da Silva ${ }^{5}$, Mateus da Silva Junqueira ${ }^{6}$, Felipe Machado Trombete ${ }^{7 *}$
}

\begin{abstract}
Resumo
O caldo-de-cana é uma bebida obtida do esmagamento da cana-de-açúcar através de moendas, geralmente acrescido de gelo e frutas cítricas, muito apreciado pelos consumidores e, geralmente, comercializada em feiras livres e por ambulantes em vias públicas. Para que a obtenção do caldo-de-cana seja feita de forma segura é fundamental a adoção de Boas Práticas relacionada com o beneficiamento, preparo, armazenamento e distribuição da bebida. Esse trabalho teve como objetivo quantificar matérias estranhas e sujidades totais em amostras de caldo-de-cana comercializadas em Sete Lagoas-MG. Foram coletadas 21 amostras da bebida e analisadas pelo método de filtração direta, seguido de observações em microscópio estereoscópio e microscópio ótico acoplados com câmera digital. Do total de amostras analisadas, $61,9 \%$ apresentaram matérias estranhas indicativas de falhas de boas práticas e 14,3\% apresentaram matérias estranhas indicativas de riscos à saúde humana. Com relação às análises físico-químicas, verificou-se que a acidez expressa em ácido cítrico variou de 0,05 a $0,18 \%$, o pH de 5,5 a 6,17 e o teor de sólidos solúveis de 16,4 a $28,6^{\circ}$ Brix. Pode-se concluir que apenas $23,8 \%$ estavam adequadas para consumo. As principais falhas verificadas no momento da coleta que podem explicar tal contaminação foram a exposição da cana-de-açúcar no ambiente com presença de vetores, falta de proteção para cabelos dos manipuladores, bem como o contato das roupas do manipulador com a matéria-prima e o descarte incorreto dos resíduos sólidos gerados no processamento.
\end{abstract}

Palavras-chave: Microscopia de Alimentos. Garapa. Avaliação da qualidade. Feiras livres.

\section{Determination of extraneous matters and physicochemical analysis in sugarcane juice marketed in the region of Sete Lagoas - MG.}

\author{
Abstract \\ ${ }^{1}$ Universidade Federal de São João del-Rei, Sete Lagoas-MG, Brasil \\ https://orcid.org/0000-0002-3806-5833 \\ ${ }^{2}$ Universidade Federal de São João del-Rei, Sete Lagoas-MG, Brasil \\ https://orcid.org/0000-0002-5265-7683 \\ ${ }^{3}$ Universidade Federal de São João del-Rei, Sete Lagoas-MG, Brasil \\ https://orcid.org/0000-0002-8923-545X \\ ${ }^{4}$ Universidade Federal de São João del-Rei, Sete Lagoas-MG, Brasil \\ https://orcid.org/0000-0002-9518-5911 \\ ${ }^{5}$ Universidade Federal de São João del-Rei, Sete Lagoas-MG, Brasil \\ https://orcid.org/0000-0003-3116-5070 \\ ${ }^{6}$ Universidade Federal de São João del-Rei, Sete Lagoas-MG, Brasil \\ https://orcid.org/0000-0002-6878-9226 \\ ${ }^{7}$ Universidade Federal de São João del-Rei. Sete Lagoas, MG. \\ https://orcid.org/0000-0002-8590-4142
}

Sugarcane juice, or garapa, is a beverage obtained from the crushing of sugarcane, usually added of citrus fruit, very appreciated by Brazilian consumers and usually marketed in open markets and by street vendors. The adoption of good practices related to the processing, preparation, storage and distribution of garapa is of great importance to produce a safe product, under adequate hygienic conditions. This work aimed to quantify extraneous matter and filth in sugarcane juice marketed in Sete Lagoas-MG. Twenty-one samples were analyzed by the direct filtration method, followed by observations in a stereomicroscope and optical microscope coupled with a digital camera. Of the total samples analyzed, 61,9\% presented extraneous

Recebido para publicação em 28 de abril de 2019. Aceito para publicação em 09 de agosto de 2019.

e-ISSN: 2447-6218 / ISSN: 2447-6218 / (c) 2009, Universidade Federal de Minas Gerais, Todos os direitos reservados. 
Rodrigues, E. D. et al.

matters indicating failures on good practice and $14,3 \%$ presented extraneous matters indicative of risks to human health. Regarding the physicochemical analyzes, it was verified that the acidity expressed in citric acid ranged from 0.05 to $0.18 \%$, pH from 5.5 to 6.17 and the soluble solids content from 16.4 to $28.6^{\circ}$ Brix. It can be concluded that only $23.8 \%$ of samples can be considered adequate for consumption. The main flaws detected that may explain such contamination were the exposure of sugarcane in the environment with presence of vectors, food handlers without protection for hair, as well as the contact of their clothes with the raw material and incorrect disposal of solid wastes generated during processing.

Key words: Food Microscopy. Garapa. Quality assessment.

\section{Introdução}

O caldo-de-cana, também conhecido como garapa, é uma bebida obtida do esmagamento da cana-de-açúcar através de moendas, geralmente acrescido de gelo e frutas cítricas. É considerada uma bebida energética, com cerca de $18,2 \%$ de sacarose, com destaque também para os minerais magnésio $(12,5 \mathrm{mg} / 100 \mathrm{~g})$, cálcio $(9,1$ $\mathrm{mg} / 100 \mathrm{~g})$ e vitamina C $(2,8 \mathrm{mg} / 100 \mathrm{~g})$ (TACO, 2011). A comercialização do caldo-de-cana em vias públicas e em feiras municipais é tradição em muitas cidades, já que é uma bebida apreciada por muitos consumidores, principalmente em épocas mais quentes do ano.

Na cidade de Sete Lagoas-MG, Silva et al. (2018a) verificaram que em um grande número de feiras-livres são comercializados desde produtos de artesanato a alimentos in natura e bebidas prontas para o consumo. Em tais feiras, muitos manipuladores não possuem informações adequadas sobre Boas Práticas de Manipulação e as Boas Praticas de Fabricação, o que pode comprometer a segurança dos produtos alimentícios comercializados. São recorrentes também, na produção do caldo-de-cana, práticas inadequadas tais como o armazenamento da cana-de-açúcar em contato direto com piso, limpeza deficiente das moendas, inexistência de uniformes adequados, acúmulo de bagaço com consequente atração de vetores, além de assepsia das mãos incorreta ou mesmo inexistente (Caldas et al., 2018). Todas essas falhas nas boas práticas podem acarretar a contaminação do produto por agentes biológicos, químicos e físicos.

De acordo com a Agência Nacional de Vigilância Sanitária (ANVISA), através do Regulamento Técnico de Procedimentos Higiênico-Sanitários para Manipulação de Alimentos e Bebidas Preparados com Vegetais (Brasil, 2005), para que a obtenção do caldo-de-cana seja feita de forma segura, em condições adequadas de higiene, é fundamental a adoção de Boas Práticas relacionados com o beneficiamento, preparo, armazenamento e distribuição da bebida. A qualidade desse produto e as deficiências em tais procedimentos têm sido verificadas por autores de diferentes localidades, por meio principalmente de análises microbiológicas, físico-químicas e microscópicas (Prado et al., 2010; Brezovsky et al., 2016; Sprenger et al., 2016).

A microscopia de alimentos é uma técnica microanalítica que pode ser utilizada como ferramenta de controle de qualidade de bebidas e objetiva identificar principalmente matérias estranhas físicas e sujidades (fragmentos de origem animal), permitindo constatar se o material analisado está adequado em relação à legislação (Santos et al. 2017; Barbieri, 2001). As matérias estranhas são definidas como qualquer material não constituinte do produto, associado a condições ou práticas inadequadas, sendo classificadas pela atual legislação sobre microscopia de alimentos em dois grupos: i) indicativas de riscos à saúde humana, capazes de veicular agentes e/ou causar danos ao consumidor, abrangendo as formigas, baratas, roedores, pombo, morcego e, ii) indicativas de falhas das Boas Práticas, abrangendo os artrópodes, pelos humanos, fungos filamentosos, areia, terra, entre outros (Brasil, 2014).

O objetivo do presente trabalho foi quantificar matérias estranhas e sujidades totais em amostras de caldo-de-cana comercializadas em feiras livres, vias públicas e estabelecimentos da região de Sete Lagoas-MG.

\section{Material e métodos}

\section{Amostragem e local das análises}

Foram coletadas, aleatoriamente, 21 amostras de caldo-de-cana in natura comercializadas na região de Sete Lagoas/MG, em diferentes pontos de venda, tais como feiras livres $(n=7)$, vias públicas $(n=6)$ e estabelecimentos comerciais $(n=8)$. Para cada ponto de coleta uma amostra foi adquirida. Não foram utilizados critérios para a escolha dos pontos de venda da bebida. Para a coleta, foram utilizadas garrafas plásticas de polietileno com tampa rosqueável, previamente lavadas e secas. Foi solicitado ao vendedor $300 \mathrm{~mL}$ da bebida, o que correspondente a um copo pequeno (opção mais comum de consumo nas feiras). O produto foi então transportado até o Laboratório de Preparo Microscópico (LPM), do Departamento de Engenharia de Alimentos (DEALI) da UFSJ e congelado a $-15^{\circ} \mathrm{C}$ até o momento das análises.

\section{Check list para verificação das condições higiênicas nos pontos de venda}

Baseando-se na RDC ㄲo 218/2005 da ANVISA (Brasil, 2005), realizou-se uma pesquisa das condições higiênicas nos pontos de aquisição das amostras, na forma de cliente oculto, utilizando um questionário que foi preenchido pela pesquisadora após adquirir a amostra. 
Pesquisa de matérias estranhas e avaliação físico-química de caldo-de-cana comercializado na região de Sete Lagoas - MG

Esta pesquisa teve como objetivo avaliar os aspectos da cana-de-açúcar e do manipulador, tais como: condições de armazenamento da cana, se eram descascadas ou não para a extração do caldo; local de destinação do bagaço; se o manipulador fazia uso de proteção para cabelo e barba; uso de avental/jaleco; presença de adornos; presença de vetores nas proximidades, dentre outros. Todas as observações foram realizadas a fim de relacionar tais informações com os resultados obtidos posteriormente nas análises microscópicas.

\section{Análise microscópica}

As matérias estranhas foram isoladas das amostras por filtração direta em papel filtro, de acordo com os procedimentos descritos no método 945.75 da Association of Official Analytical Chemists (AOAC, 2016), conforme descrito a seguir. As amostras de caldo-de-cana foram descongeladas por cerca de $2 \mathrm{~h}$ em temperatura ambiente e todo o conteúdo foi filtrado através de papel filtro qualitativo (125 mm, Unifil), com auxílio de vácuo (bomba Marconi MA059). A primeira parte do material filtrado foi destinada as análises físico-químicas. Todo o material em contato com o caldo-de-cana foi lavado utilizando jatos de água destilada, objetivando coletar todos os fragmentos que poderiam permanecer no frasco. Essa segunda filtragem foi desprezada e o filtro de papel contendo os fragmentos retidos foi transferido para uma placa de Petri, identificado e congelado até o momento das observações microscópicas.

Para a identificação dos fragmentos retidos no filtro foi utilizado um microscópio estereoscópio (ZEISS Primo Star), e um microscópio ótico (ZEISS Stemi 2000-C) ambos acoplados com câmera (ZEISS Axiocam ERc5s). Inicialmente, procedeu-se a observação no estereoscópio com aumento de 7 vezes. Os fragmentos foram então coletados com auxílio de palito de madeira e glicerina, sendo transferidos para lâminas contendo água destilada e cobertos com lamínula, a fim de confirmação no microscópio ótico (quando necessário), utilizando aumento de 40, 100 e 400 vezes. A identificação dos fragmentos de insetos, pelos e demais matérias estranhas e sujidades basearam-se nas referências de Barbieri (2001) e Oliveira et al. (2015).

\section{Análise físico-química}

As amostras foram analisadas quanto aos seguintes parâmetros físico-químicos: $\mathrm{pH}$, acidez total titulável e teor de sólidos solúveis, utilizando metodologias propostas pelo Instituto Adolfo Lutz (IAL, 2008). O pH foi verificado com auxílio de um pHmetro (mPA210 MS Tecnopon), previamente calibrado com soluções tampão 4,0 e 7,0, através da imersão direta do eletrodo na amostra (método 17/IV). O teor de sólidos solúveis foi verificado através da amostra colocada sobre o prisma de um refratômetro (AtagoPAL-2), conforme método 315/ IV. A acidez total titulável foi determinada conforme o método 253/IV, utilizando $10 \mathrm{~mL}$ de amostra e $30 \mathrm{~mL}$ de água destilada, sendo então adicionadas 3 gotas de fenolftaleína e titulado com solução de $\mathrm{NaOH}(0,1 \mathrm{M})$. $\mathrm{O}$ ponto de viragem foi verificado quando a amostra apresentou $\mathrm{pH}$ entre 8,2-8,4. O volume de $\mathrm{NaOH}(\mathrm{mL})$ gasto na titulação foi anotado e a acidez foi expressa em $\mathrm{g}$ de ácido cítrico/100 $\mathrm{mL}$ da bebida, conforme o método 312/IV, utilizando a fórmula abaixo:

\section{V.F.M.PM \\ $\frac{10 . P . N}{\text { 10. }}=$ GDE ÁCIDO CÍTRICO/100 ML (EQ. 1)}

Onde:
$\mathrm{V}=$ volume da solução de $\mathrm{NaOH} 0,1 \mathrm{M}$ em mL
$\mathrm{M}=$ molaridade da solução de $\mathrm{NaOH}$
$\mathrm{P}=$ volume pipetado da amostra em $\mathrm{mL}$
$\mathrm{PM}=$ peso molecular do ácido cítrico $(192 \mathrm{~g})$
$\mathrm{N}=$ número de hidrogênios ionizáveis $(\mathrm{n}=3)$
$\mathrm{F}=$ fator de correção da solução de hidróxido de sódio

\section{Resultados e Discussão}

Por meio da pesquisa por cliente oculto foram observadas importantes falhas de higiene na manipulação da cana-de-açúcar, bem como durante e seu processamento. Em 26,6\% dos pontos verificados a cana não apresentava-se limpa ou lavada e em 86,7\% não estava armazenada em local apropriado, como por exemplo, sobre prateleiras ou paletes impermeável e lavável, protegido de vetores e pragas, conforme determina a legislação. Em 93,33\% dos pontos visitado a cana-de-açúcar não era descascada antes de ser moída, e 100\% dos manipuladores não descascava a cana fora do local de trabalho. Mais da metade dos vendedores $(53,33 \%)$ não utilizava cabelos protegidos por toucas, rede ou boné, o que pode acarretar na presença de pelos, tanto na matéria-prima quanto já na bebida pronta. Em 20\% dos locais visitados os manipuladores possuíam barba desprotegida. Foi observado também que $66,7 \%$ dos vendedores que manipularam a cana também receberam o pagamento em dinheiro, sem realizar posteriormente algum procedimento de assepsia.

A moenda aparenta estar em boas condições de limpeza e higiene (ferrugem, sujeira, graxa aparente) nos locais visitados $(86,67 \%)$, outro ponto importante verificado foi a presença de animais nas proximidades dos pontos de coleta das amostras, onde, em cerca de metade dos locais visitados $(53,3 \%)$ foi observada principalmente a presença de pombos, moscas e abelha arapuã (Trigona spinipes). A maioria (60\%) também não possuía local para lavagem das mãos .Verificou-se também 93,3\% dos locais é comum o acúmulo de bagaço nas proximidades do local de processamento, oferecendo condições para atração de vetores, uma vez que a maioria dos comerciantes os descarta apenas uma vez por dia e no final do expediente. Em 86,7\% não utilizavam uniformes ou proteções como aventais e jalecos e o uso de anéis, brincos e relógios foi verificado em 93,3\% dos manipuladores. 
Rodrigues, E. D. et al.

Outro ponto importante observado é o armazenamento do caldo residual que sobra entre as vendas, o qual é mantido sem refrigeração e misturado com o caldo da nova extração. Esse fato também foi verificado por Caldas et al. (2018) e, juntamente com as demais falhas observadas na presente pesquisa, podem constituir em grave problema de saúde pública, uma vez que o caldo-de-cana é um produto de alta atividade de água, rico em açúcares e oferece excelente meio para crescimento de micro-organismos deteriorantes e patogênicos (Silva et al., 2018b), somado ao fato de ter alto consumo e aceitação pelos consumidores.

Felipe e Miguel (2011) e Gassen et al. (2017) também relataram problemas semelhantes aos verificados no presente estudo, tais como cabelos desprotegidos, utilização de adornos e ausência de locais para lavagem das mãos, demonstrando serem problemas recorrentes em diferentes localidades.

As inconformidades com a legislação observadas no check list durante a pesquisa por cliente oculto justificam a presença dos fragmentos encontrados na análise microscópica (Figura 1). Das 21 amostras avaliadas, em $16(76,2 \%)$ foram encontradas matérias estranhas como fibras de tecidos e fungo filamentoso, além de sujidades representadas principalmente por fragmentos de insetos, insetos inteiros, pelos e bárbulas de pombo. Em uma mesma amostra foram encontrados fragmentos de insetos, pelo e fibras de tecido, indicando total falha nas Boas Práticas. Em outra amostra foram encontrados dois pelos humanos e, nesse mesmo ponto de coleta, foi observado que o manipulador não fazia uso de proteção nos cabelos (Figura 2A), o que pode justificar tal fato. Também, foi verificada a presença de bárbulas de pombo em uma amostra, sendo evidenciada no ponto de coleta de tal produto a presença de pombos próximos ao local de processamento da bebida (Figura 2B), nesse mesmo local, também verificou-se que a roupa do manipulador estava em contato direto com a cana-de-açúcar, o que pode justificar a presença de 9 fragmentos de fibra de tecido na mesma amostra. Em outra amostra foi encontrada um pelo, um fragmento de inseto e dez matérias estranhas, no ponto de coleta foi observado que o manipulador não usava roupas apropriadas e o descarte do bagaço era feito direto no chão. Foi encontrado em uma mesma amostra quatro formigas inteiras e 2 fragmentos de formigas, representando um risco a saúde.

Em outra amostra foi encontrada uma formiga inteira, além de fibras de tecidos. A presença de fragmentos de insetos também foi verificada em uma amostra onde o caldo-de-cana era comercializado em uma rodovia, embaixo de árvores e o descarte do bagaço era feito no chão do próprio local, fatores estes que favorecem a aproximação de insetos (Figura 2C). A presença de insetos como as formigas, pode passar a falsa impressão de não representar um perigo. No entanto, conforme apresentado por Fontana et al. (2010) e Sousa et al. (2016), tais insetos são vetores de várias bactérias, tais como Escherichia coli, Arizona ssp., Enterobacter spp., Klebsiella spp, Hafnia spp., Yersinia enterocolitica e Staphylococcus Coagulase Negativa, Staphylococcus aureus Coagulase Positiva, Staphylococcus epidermidis, dentre outros, que podem causar importantes doenças.

Figura 1 - Imagens obtidas na análise microscópica de amostras de caldo-de-cana comercializadas na região de Sete Lagoas - MG.
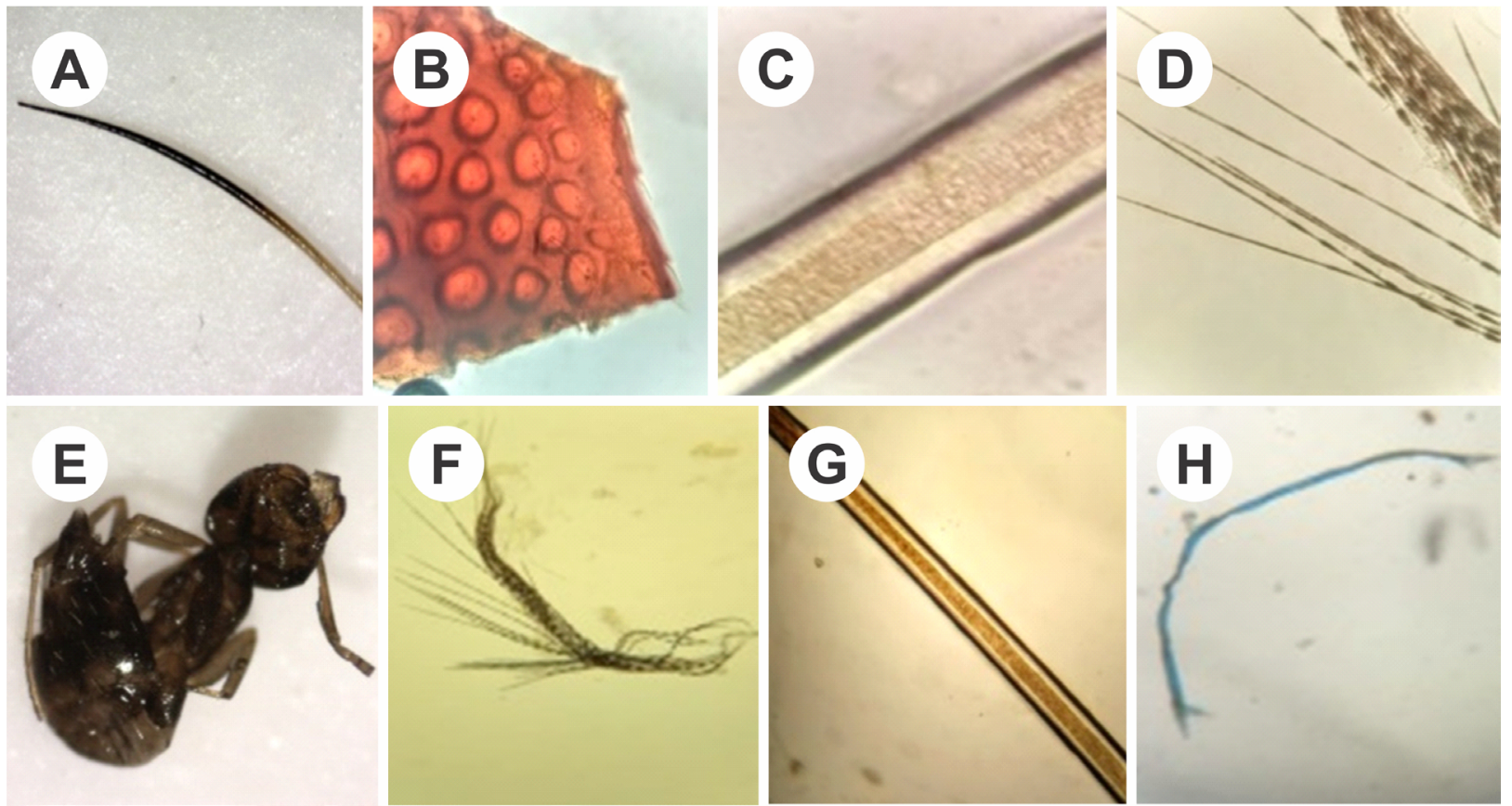

Identificação: A: Pelo (aumento de 50x). B: Fragmento de inseto (100 x). C: Pelo (400 x). D: Bárbula de pombo (100 x). E: Formiga (50x). F: Bárbula de pombo (40x). G- Pelo (100 x). H: Fibra de tecido (100x). 
Pesquisa de matérias estranhas e avaliação físico-química de caldo-de-cana comercializado na região de Sete Lagoas - MG

De acordo com o Regulamento Técnico de Procedimentos Higiênico-Sanitários para Manipulação de Alimentos e Bebidas Preparados com Vegetais (Brasil, 2005), quaisquer substâncias ou agentes de origem biológica, química ou física, estranhos às bebidas que sejam considerados nocivos à saúde humana ou que comprometam a sua integridade são considerados contaminantes. Dessa forma, pode-se afirmar que pelos resultados obtidos das avaliações microscópicas, apenas $23,80 \%$ das amostras podem ser consideradas adequadas para consumo, não representando um risco para a saúde ou falhas nas boas práticas. Em 61,90\% foram encontradas matérias estranhas indicativas de falhas de boas práticas e em 14,30\% foram encontradas matérias estranhas indicativas de riscos à saúde humana.

Outros autores também relataram problemas relacionados com a obtenção higiênica do caldo-de-cana. Em um estudo realizado por Prado et al. (2010), na cidade de Ribeirão Preto - SP, foi verificada a presença de diferentes tipos de sujidades, tais como asas, patas e antenas de insetos, além de contaminação por pelos de roedores e fibras sintéticas. Sprenger et al. (2016) também relataram a presença de fibras sintéticas nas amostras de caldo-de-cana, representando falhas nas boas práticas de produção.

Figura 2 - Coleta das amostras de caldo-de-cana comercializado no município de Sete Lagoas - MG.
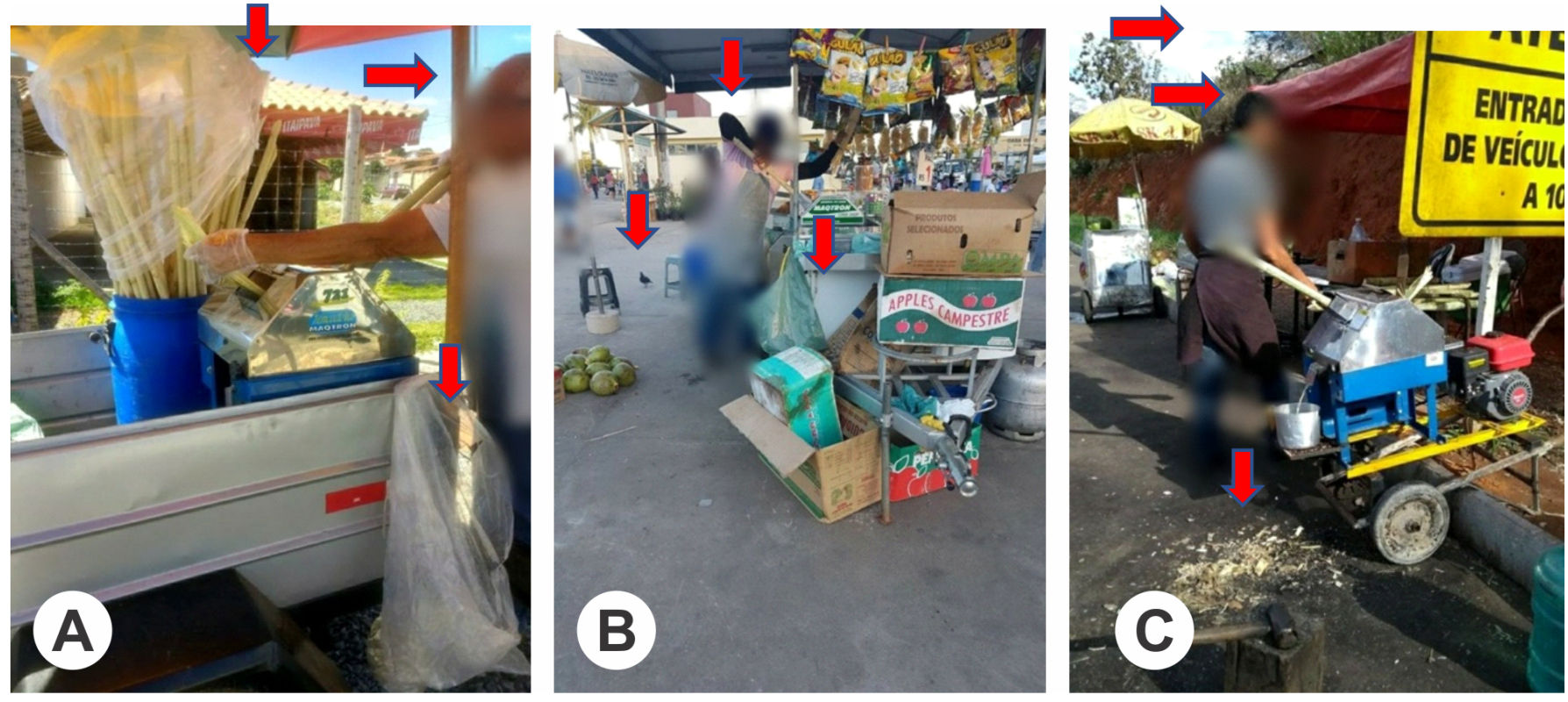

Inconformidades verificadas: A- Cabelo sem proteção, cana-de-açúcar desprotegida, saco com lixo próximo a moenda; B - Presença de pombo, roupa do manipulador em contato direto com a cana-de-açúcar, acúmulo de lixo próximo a moenda; C - Cabelo sem proteção, acumulo de resíduos próximos a moenda, comercialização em local aberto coberto por árvores.

Com relação as análises físico-químicas, verificou-se que a acidez expressa em ácido cítrico variou de 0,05 a $0,18 \%$, o pH de 5,5 a 6,17 e o teor de sólidos solúveis de 16,4 a $28,6^{\circ}$ Brix. Tais valores são semelhantes aos reportados por Kunitake (2012) e Andrade (2014), que encontraram para caldo-de-cana fresco acidez de 0,04 a 0,07\% (em ácido cítrico), pH entre 5,0 a 5,6; e sólidos solúveis de 15,2 a $23,6^{\circ}$ Brix. A grande variação no teor de açúcares presentes nos caldo-de-cana avaliados pode ser explicada pelo fato de não ser um produto padronizado, sendo utilizadas cultivares diferentes de cana-de-açúcar para a extração do caldo e também com diferente grau de maturação. Não existem parâmetros físico-químicos para a qualidade do caldo-de-cana e, no presente estudo não foi possível associar tais resultados com aqueles das análises microscópicas e com os obtidos no check list.

\section{Conclusão}

Das 21 amostras de caldo-de-cana in natura comercializadas na região de Sete Lagoas/MG, 61,9\% apresentaram matérias estranhas indicativas de falhas de boas práticas e 14,3\% apresentaram matérias estranhas indicativas de riscos à saúde humana. Do total de amostras analisadas, apenas $23,8 \%$ podem ser consideradas adequadas para consumo. As principais falhas verificadas no momento da coleta das bebidas foram a exposição da cana-de-açúcar no ambiente com presença de vetores, falta de proteção para cabelos dos manipuladores, bem como o contato das roupas do manipulador com a matéria-prima e o descarte incorreto dos resíduos sólidos gerados no processamento. Com relação às análises físico-químicas, verificou-se que os valores obtidos foram característicos da bebida. É importante ressaltar a necessidade da realização de programas de capacitação e treinamento com tais manipulares, como forma de orientar as práticas de higiene e técnicas adequadas de manipulação, garantindo a inocuidade do caldo-de-cana e, consequentemente, prevenindo a contaminação do produto. 
Rodrigues, E. D. et al.

\section{Referências}

Andrade, I. M. G. 2014. Estimativa da vida de prateleira de caldo de cana padronizado estocado sob refrigeração. 2014. 162 f. Dissertação (Mestrado em Engenharia de Alimentos) - Faculdade de Zootecnia e Engenharia de Alimentos, Universidade de São Paulo, Pirassununga.

AOAC. 2016. Association of Official Analytical Chemists. Official Methods of Analysis of AOAC International, 20th Edition, Gaithersburg, USA.

Barbieri, M.K.2001. Microscopia em alimentos: identificação histológica e material estranho. ITAL. São Paulo.

Brasil. 2014. Anvisa agência nacional de vigilância sanitária. Resolução da diretoria colegiada - RDC № 14, DE 28 DE MARÇO DE 2014. Disponível em :http://bvsms.saude.gov.br/bvs/saudelegis/anvisa/2014/ rdc0014_28_03_2014.pdf

Brasil. 2005. Anvisa agência nacional de vigilância sanitária. RDC no 218, de 29 de julho de 2005. Regulamento Técnico de Procedimentos Higiênico-Sanitários para Manipulação de Alimentos e Bebidas Preparados com Vegetais. Disponível em: http://portal.anvisa.gov.br/ documents/33916/388704/RDC_218.pdf

Brezovsky, F. R.; Valiatti, T. B.; Romão, N. F.; Passoni, G. P.; Sobral, F. D. O. S. 2016. Avaliação Microbiológica e Microscópica do Caldo de Cana Comercializado em Ji-Paraná. Ensaios e Ciência: Ciências Biológicas, Agrárias e da Saúde 20:2. DOI: http://dx.doi.org/10.17921/14156938.2016v20n2p111-115

Caldas, S. H.; Batista, A.A.; Assunção, A. P.; Mendes, A. C. 2018. Avaliação das Condições de Comercialização e Frequência de Consumo de Caldo de Cana pela População de Salvador-BA. PLURAIS-Revista Multidisciplinar 1: 2. DOI: http://dx.doi.org/10.29378/plurais.2447-9373.2010. v1.n2.\%25p

Felipe, L. M.; Miguel, D. P. 2011. Análise da qualidade microbiológica do caldo-de-cana. FAZU em Revista, Uberaba 8: 77-82. Disponível em: http://www.fazu.br/ojs/index.php/posfazu/article/view/410.

Fontana, R.; Wetler, R. D. C.; Aquino, R. S., Andrioli, J. L.; Queiroz, G. R.; Ferreira, S. L.; Delabie, J. H. 2010. Disseminação de bactérias patogênicas por formigas (Hymenoptera: Formicidae) em dois hospitais do nordeste do Brasil. Neotrop Entomol 39: 655-663.

Gassen, S.G.; Peder,L.D.; Silva,C.M. 2017. Análise da qualidade microbiológica do caldo de cana comercializado em um município da região oeste do paraná. In: Colloquium Vitae 9: 7-12.
Instituto Adolfo Lutz. 2008. Métodos físico-químicos para análise de alimentos. Ribeirão Preto: Instituto Adolfo Lutz 1020. São Paulo.

Kunitake, M. T. 2012. Processamento e estabilidade de caldo de cana acidificado. 2012. 131 f. Dissertação (Mestrado em Engenharia de Alimentos) - Faculdade de Zootecnia e Engenharia de Alimentos, Universidade de São Paulo, Pirassununga.

Oliveira, F.; Jorge, L.I.F; Ritto, J.L.A.; Barroso, I.C.E.; Prado, B.W. 2015. Microscopia de Alimentos: Exames Microscópicos de Alimentos in Natura e Tecnologicamente Processado. p.412. Atheneu. São Paulo.

Prado, S. D. P. T.; Bergamini, A. M. M.; Ribeiro, E. G. A.; Castro, M. D. C. S.; De Oliveira, M. A. 2010. Avaliação do perfil microbiológico e microscópico do caldo de cana in natura comercializado por ambulantes. Rev. Inst. Adolfo Lutz 69: 55-61.

Santos, P. C.; Souza, E. M.; Lopes, L. C.; Vieira, M. C.; Cunha, L. R.; Lima, E. M. F. 2017. Avaliação microscópica e pesquisa de sujidades em amostras comerciais de orégano (Origanum vulgare L.), salsa (Petroselinum sativum Hoffim.) e chimichurri. Cad. Ciênc. Agrá. 09: 11-16. Disponível em: https://periodicos.ufmg.br/index.php/ccaufmg/ article/view/2992.

Silva, A. N. A.; Parada, A. D.; Neves, L. M.; Cury, J. C., Silva, A. M. 2018a. Capacitação de manipuladores de alimentos das feiras livres do município de Sete Lagoas - MG. XVI Congresso de Produção Científica e Acadêmica. XVI SEMEX - Semana de Extensão Universitária. Universidade Federal de São João del-Rei. Sete Lagoas.

Silva, D. A.; Martins, L. T. S.; Soares, L. S. 2018b. Avaliação microbiológica do caldo de cana comercializado por ambulantes e estabelecimentos formais em Salvador, BA. Hig. Alimente 32: 80-84.

Sousa, L. F.; Oliveira, B. R. M.; Soares, R. C.; Nascimento, T. C.; Madureira, M. S.; Fortua, J. L. 2016. Pesquisa de enterobactérias e estafilococos em formigas em ambiente hospitalar. Ciência \& Tecnologia Fatec-JB 8.

Sprenger, L.K.; Risolia, L.W.; Hamdar, S.Z.; Molento, M.B. 2016. Análise microbiológica de caldos de cana comercializados em Curitiba, Paraná. Archives of Veterinary Science. ISSN 1517-784X 21: 01-07. DOI: http:// dx.doi.org/10.5380/avs.v21i4.38829.

Taco. 2011. Tabela Brasileira de Composição de Alimentos: 4⿳亠 edição revisada e ampliada. Núcleo de Estudos e Pesquisas em Alimentação (NEPA). Universidade Estadual de Campinas (UNICAMP). 2011. 